\title{
Identification of mesenchymal stromal cells in human lung parenchyma capable of differentiating into aquaporin 5-expressing cells
}

\author{
Golnaz Karoubi, Lourdes Cortes-Dericks, Isabel Breyer, Ralph A Schmid and André E Dutly
}

The lack of effective therapies for end-stage lung disease validates the need for stem cell-based therapeutic approaches as alternative treatment options. In contrast with exogenous stem cell sources, the use of resident progenitor cells is advantageous considering the fact that the lung milieu is an ideal and familiar environment, thereby promoting the engraftment and differentiation of transplanted cells. Recent studies have shown the presence of multipotent 'mesenchymal stem cells' in the adult lung. The majority of these reports are, however, limited to animal models, and to date, there has been no report of a similar cell population in adult human lung parenchyma. Here, we show the identification of a population of primary human lung parenchyma (pHLP) mesenchymal stromal cells (MSCs) derived from intraoperative normal lung parenchyma biopsies. Surface and intracellular immunophenotyping by flow cytometry revealed that cultures do not contain alveolar type I epithelial cells or Clara cells, and are devoid of the following hematopoietic markers: CD34, CD45 and CXCR4. Cells show an expression pattern of surface antigens characteristic of MSCs, including CD73, CD166, CD105, CD90 and STRO-1. As per bone marrow MSCs, our pHLP cells have the ability to differentiate along the adipogenic, osteogenic and chondrogenic mesodermal lineages when cultured in the appropriate conditions. In addition, when placed in small airway growth media, pHLP cell cultures depict the expression of aquaporin 5 and Clara cell secretory protein, which is identified with that of alveolar type I epithelial cells and Clara cells, respectively, thereby exhibiting the capacity to potentially differentiate into airway epithelial cells. Further investigation of these resident cells may elucidate a therapeutic cell population capable of lung repair and/or regeneration.

Laboratory Investigation (2009) 89, 1100-1114; doi:10.1038/labinvest.2009.73; published online 3 August 2009

KEYWORDS: cell therapy; differentiation; human lung; lung parenchyma cells; mesenchymal stem cells; stem cell therapy

Currently, the best treatment option for end-stage lung disease is transplantation with a mean 5-year survival rate of just above $50 \%{ }^{1}$ It is at most only an adequate means of therapy with poor results, high morbidity rates and donor shortage limitations. It is therefore imperative to explore new therapeutic strategies for repair and regeneration of damaged lung. ${ }^{2}$ Ideally, a reparative or regenerative treatment not involving total organ replacement requiring life-long immunosuppression would be desirable. One such approach is regenerative cell therapy using stem or progenitor cells. Stem cell-based approaches involve the in vitro expansion and/or differentiation of embryonic, fetal, amniotic, umbilical cord blood or adult stem cells into functional progeny with subsequent transplantation. ${ }^{3}$
Adult stem cells, found in select niches in developed organs, are believed to actively participate in replenishment of mature cell types within their respective organ or tissue., ${ }^{3,4}$ Generally, they are believed to be constrained to their tissue of origin with an ability to generate only the mature cell type(s) pertaining to that specific tissue. This view, however, has been challenged by a surge of studies showing the potential of adult stem and/or progenitor cells to engraft into various organs and differentiate accordingly, in response to the local milieu. ${ }^{2,5-10}$ The lung is a very complex organ, and to maintain proper working function, it requires the cooperative function of over 40 different cell types. The anatomical and functional complexity of the lung ${ }^{11,12}$ impedes, at least in part, the progress in the study of stem

Division of General Thoracic Surgery, University Hospital Berne, Berne, Switzerland

Correspondence: Professor RA Schmid, MD, Division of General Thoracic Surgery, University Hospital Berne, Berne CH-3010, Switzerland.

E-mail: ralph.schmid@insel.ch

Received 28 December 2008; revised 13 May 2009; accepted 13 May 2009 
cells. Furthermore, this complexity results in a lack of adequate cell sources for cell-based therapeutic approaches, which are both exogenous in nature as well as from within the lung itself. To date, a single lung stem cell capable of differentiating into all the cellular components of the lung has not been identified. By contrast, each region of the adult organ seems to be maintained by its own stem or progenitor cell population. ${ }^{4,13-18}$

To date, cell-based therapy approaches to the lung have focused on the use of non-resident stems cells, especially concentrating on cells located in the bone marrow ${ }^{5-8,19-25}$, and more specifically, on the mesenchymal stromal cell (MSC) population, hence termed as bone marrow MSCs (BMSC). MSCs are cells of stromal origin with the ability to self-renew and differentiate into a number of cell lineages. They were first described in a population of bone marrow cells and since then have been identified in a wide variety of tissues, including umbilical cord blood, Wharton's jelly, placenta and adipose tissue. ${ }^{26-31}$ MSCs are devoid of hematopoietic and endothelial cell markers; express variable levels of CD73, CD90, CD105, CD166 and STRO-1; and undergo differentiation to adipocytes, osteoblasts and chondrocytes. ${ }^{32-35}$

In the past decade, several publications ${ }^{6-8,19-25}$ have shown the capacity of BMSCs in engrafting and assuming lung phenotypes after transplantation. These studies, however, have been plagued with inconsistent results and controversy as to whether adult bone marrow stromal cells really engraft and differentiate into lung phenotypes. ${ }^{23,36}$ The present consensus seems to be that engraftment of bone marrow-derived MSCs in the lung does occur, but at the very low rates of approximately $0.01-0.1 \%,{ }^{36,37}$ and hence they are unlikely to be a practical option for cell-based therapy studies.

In contrast to exogenous stem cell sources, the use of resident progenitor cells as a therapeutic population is advantageous given that the lung milieu is an ideal and familiar environment, hence promoting the engraftment, survival and regenerative capacity of the transplanted cells. Recent studies have indicated the presence of 'mesenchymallike' progenitor cells in the lung with multipotent capabilities. ${ }^{3-42}$ The majority of these studies, however, are limited to animal models and no one has yet identified an analogous population in adult human lung parenchyma. In this report, we describe the isolation and characterization of a previously unidentified subset of cells exhibiting MSC properties, derived from normal human lung parenchyma. These cells portray progenitor cell characteristics and may potentially be investigated for use in cell therapy applications on the lung.

\section{MATERIALS AND METHODS Isolation and Culture of Human Lung Parenchyma Cell Cultures}

Biopsies were taken intraoperatively from patients undergoing lung resection. The samples were absent of any macroscopic signs of pneumonia or other parenchymatous disorders, and hence termed as 'normal' lung. Sample collection was approved by the University of Berne ethical committee and all biopsies were taken with patients' consent. Normal human lung parenchyma cell cultures were isolated using an adapted method on the basis of an animal protocol outlined in Ling et al. ${ }^{43}$ Briefly, intraoperative biopsies were collected in ice-cold phosphate-buffered saline (PBS; Invitrogen; Basel, Switzerland), cut into small pieces $(2-3 \mathrm{~mm})$ and washed in Hank's balanced salt solution (Invitrogen) containing 2\% penicillin/streptomycin (P/S; Invitrogen) solution. The minced tissue was then treated with $0.1 \%$ protease type XIV (Sigma Aldrich; Basel, Switzerland) in Joklik's minimum essential media (JMEM; Sigma Aldrich) at $4^{\circ} \mathrm{C}$ overnight. After overnight incubation, the digested product was transferred into a $50 \mathrm{ml}$ conical tube containing JMEM supplemented with $10 \%$ fetal bovine serum (FBS; Perbio Science; Lausanne, Switzerland). The mixture was pipetted several times to dislodge the pulmonary cells, and subsequently filtered through a $100-\mu \mathrm{m}$ cell strainer. The released cells were washed, and resuspended in MCDB-201 medium (Sigma Aldrich) containing insulin-transferrin-selenium supplements (Invitrogen) and $1 \% \mathrm{P} / \mathrm{S}$. Cells were plated at a density of $2.5 \times 10^{5}$ cells $/ \mathrm{ml}$ onto $\mathrm{T}_{75}$ tissue culture flasks coated with Collagen I $\left(10 \mu \mathrm{g} / \mathrm{cm}^{2}\right)$ (BD Biosciences; Basel, Switzerland). Cells were incubated overnight at $37^{\circ} \mathrm{C}$ and $5 \% \mathrm{CO}_{2}$. After $24 \mathrm{~h}$, the primary cultures were washed once with MCDB-201 medium to remove unattached cells, then replenished with MCDB-201 medium supplemented with insulin-transferrin-selenium, epidermal growth factor (Invitrogen) $(1 \mathrm{ng} / \mathrm{ml})$ and 1\% FBS. To maintain cell growth in culture, media was changed every $48 \mathrm{~h}$.

\section{Surface, Intracellular Staining and Flow Cytometry Analysis}

Adherent cells were harvested by trypsinization with $0.25 \%$ trypsin EDTA (Invitrogen) and pelleted by centrifugation at 1000 r.p.m. for $5 \mathrm{~min}$ at room temperature. Cell counts were adjusted to $10^{6}$ cells per $100 \mu \mathrm{l}$ of BD Stain Buffer (BD Biosciences). A two-step indirect staining protocol was used before flow cytometry analysis. Cells were first stained with $1 \mu \mathrm{g}$ of primary antibody: anti-human CD34 (BD Biosciences); anti-human CD73 (BD Biosciences); antihuman CD9 (BD Biosciences); anti-human CD90 (BD Biosciences); anti-human CD166 (BD Biosciences); antihuman CD105 (BD Biosciences); anti-human CXCR4 (BD Biosciences); anti-human CD45 (BD Biosciences); antihuman CD31 (BD Biosciences); anti-human STRO-1 (BD Biosciences); or the appropriate isotype control antibody: mouse IgG1 immunoglobulin isotype (BD Biosciences); and mouse IgG3 immunoglobulin isotype (BD Biosciences). The samples were then washed twice using PBS. This was followed by staining with Alexafluor 488-conjugated secondary antibody (Invitrogen) (1:500) for $30 \mathrm{~min}$ followed by two additional washes in PBS. Cells were fixed in fixation buffer (BD Biosciences) and resuspended in $300 \mu \mathrm{l}$ of stain buffer 
before analysis using the LSR II flow cytometer (BD Biosciences). For intracellular staining, cells were treated as per surface staining, but were permeabilized using cell fixation/ permeabilization buffer (BD Biosciences) before incubation with the primary antibody rabbit anti-human aquaporin 5 (AQ5; Abcam); rabbit anti-human Clara cell secretory protein (CCSP; Protein Tech Group; Manchester, UK); rabbit anti-human surfactant protein C (SPC; Santa Cruz Biotechnology; CA, USA); or rabbit IgG immunoglobulin isotype (BD Biosciences).

\section{RNA Extraction and RT-PCR}

Cell samples were collected in Trizol (Invitrogen) followed by total RNA extraction according to the manufacturer's instructions. Complementary DNA (cDNA) was synthesized by reverse transcription (RT) of 5-8 $\mu \mathrm{g}$ each of total RNA using M-MLV-RT (Promega; Wallisellen, Switzerland) and random primers (Roche Diagnostics; Rotkreuz, Switzerland). To eliminate amplification of contaminating chromosomal DNA, the isolated total RNA was treated with RNase-Free DNase (Roche Diagnostics) according to the manufacturer's instructions. Each cDNA sample was then amplified with Taq polymerase (Roche Diagnostics). The primers used for the ABCG2 and $\beta$-actin genes were: ABCG2: $5^{\prime}$-GGAACTCAGTT TATCCGTGG- $3^{\prime}$; $5^{\prime}$-CGAGGCTGATGAATGGAGAAG- ${ }^{\prime}$; and $\beta$-actin $5^{\prime}$-GAAGATGGTGATGGGGAT- $3^{\prime} ; 5^{\prime}$-GAAGGTGAAG GTCGGAGC- $3^{\prime}$. The amplified PCR products were separated on $1.5 \%$ agarose gels by electrophoresis. Digital images were captured on a Versa Doc Imaging System (Bio Rad; Reinach, Switzerland).

\section{Quantitative Real-Time RT-PCR}

mRNA expression levels of the housekeeping gene (ACTBHs_99999903_m1, $\beta$-actin) and target genes (AQ5Hs_00893081_m1, Aquaporin 5; SCP-Hs_00161628_m1, surfactant protein C; CCSP-Hs_00171092_m1; Clara cell secretory protein) were evaluated with commercially available TaqMan 'Assay on Demand' probes (Applied Biosystems; Rotkreuz, Switzerland). In total, 50 ng of resulting cDNAs were subjected to real-time RT-PCR using the specific primers and probes in a $25 \mu \mathrm{l}$ final reaction volume and analyzed in triplicate. Gene expression was detected as an amplification curve using ABI 7900 sequence detection system, and cycle number was obtained when amplification exceeded threshold ( $C_{\mathrm{t}}$ values). All target gene $C_{\mathrm{t}}$ values in each parameter were normalized by reference gene ( $\beta$-actin) $C_{\mathrm{t}}$ value to determine the $\Delta C_{\mathrm{t}}$ value (target gene $C_{\mathrm{t}}-$ reference gene $C_{\mathrm{t}}$ ). Baseline and threshold values for $C_{\mathrm{t}}$ calculation were set automatically using the ABI Prism SDS 2.1 software. The quantitative RT-PCR data represent the relative quantity of AQ5 mRNA compared with the 'Aquaporin 5 mRNA/ $\beta$-actin mRNA' ratio determined from snap-frozen normal human lung parenchyma tissue. Relative gene expression was shown as fold change (ratio) in gene expression using the comparative $C_{\mathrm{t}}$ method.

\section{Side Population Analysis}

Side population (SP) cell analysis was done as described in Goodell et $a l^{44}$ with minor modifications. Briefly, adherent cells were harvested by trypsinization, as previously described, and $1 \times 10^{6}$ cells were incubated in pre-warmed DMEM (Invitrogen) supplemented with 5\% FBS containing Hoechst 33342 (Sigma Aldrich) at a final concentration of $5 \mu \mathrm{g} / \mathrm{ml}$ in the presence or absence of $50 \mu \mathrm{M}$ of the multidrug-resistant protein inhibitor, Verapamil (Sigma Aldrich). The incubation period proceeded at $37^{\circ} \mathrm{C}$ with intermittent mixing for $90 \mathrm{~min}$. After incubation, cells were washed twice with ice-cold DMEM containing 5\% FBS, centrifuged at 1200 r.p.m. at $4^{\circ} \mathrm{C}$ for $5 \mathrm{~min}$ and resuspended in cold PBS supplemented with $1 \%$ BSA. Cells were kept on ice and analyzed immediately. Propidium iodide (Sigma Aldrich) at a final concentration of $2 \mu \mathrm{g} / \mathrm{ml}$ was added before flow cytometry-based analysis to discriminate dead cells from the viable ones. The Hoechst dye was excited using a UV laser at $351 \mathrm{~nm}$ and its fluorescence was measured using a 402/46 BP filter for Hoechst blue detection, and 675 LP filter for Hoechst red detection. A minimum of 100000 events were collected for each analysis.

\section{Immunocytochemistry and Intracellular Staining for Differentiation}

For immunocytochemistry, primary human lung parenchyma cells growing in culture were fixed in a methanol/ acetone solution (1:1) for $3 \mathrm{~min}$ at room temperature followed by three washes in PBS. Cells were permeabilized using $0.1 \%$ Triton X-100 in PBS for $5 \mathrm{~min}$ followed by a wash in PBS. Non-specific binding was blocked by incubating the cells with PBS containing 3\% BSA solution for $1 \mathrm{~h}$ at room temperature. Cells were then incubated overnight at $4^{\circ} \mathrm{C}$ with primary antibodies $(1: 200)$ or immunoglobulin isotype control antibody. This was followed by three additional washes in PBS containing 1\% BSA. Cells were incubated with Alexaflour 488-conjugated secondary antibody (1:300) for $1 \mathrm{~h}$ in dark then washed with PBS containing $1 \%$ BSA solution and analyzed using inverted fluorescence

Figure 1 Morphology of primary human lung parenchyma cells. Flow cytometry profile and phase-contrast light microscopy images of a representative primary cell culture derived from a human lung parenchyma clinical biopsy at (a) passage 0; (b) passage 1; (c) passage 3; and (d) passage 5. Images were taken at 5 and 10 days in culture at $\times 10$ magnification. Flow cytometric profiles illustrate the size (FSC, forward scatter of light) and granularity (SSC, side scatter of light) of cultures at the different passages. 
a

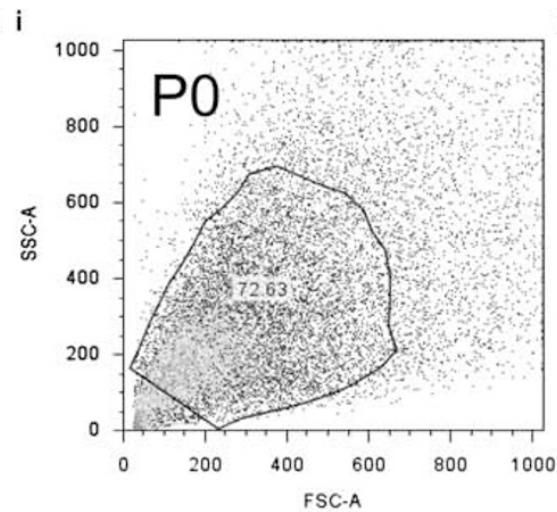

b

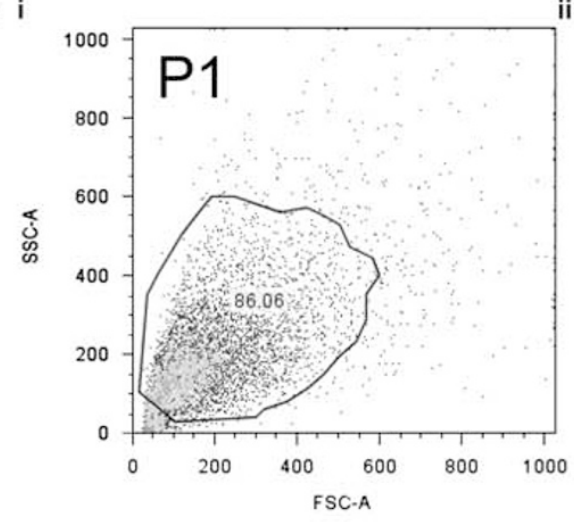

ii

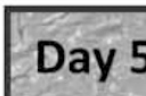

$10 x$

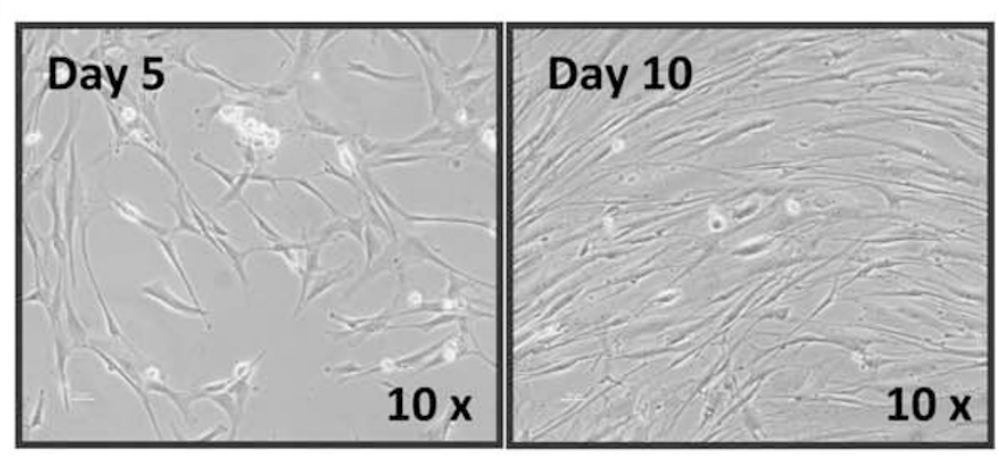

Day 10

$10 x$ c i

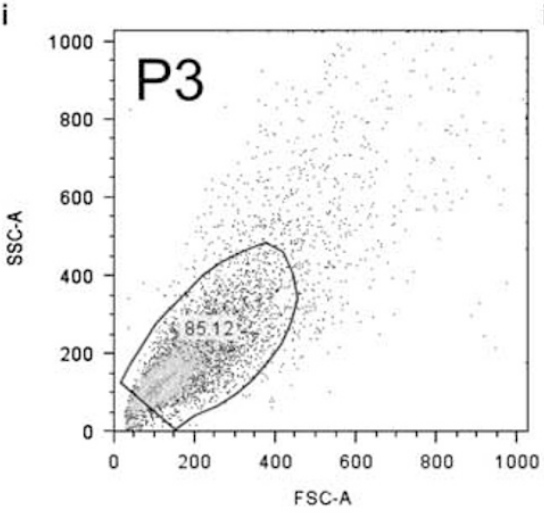

ii

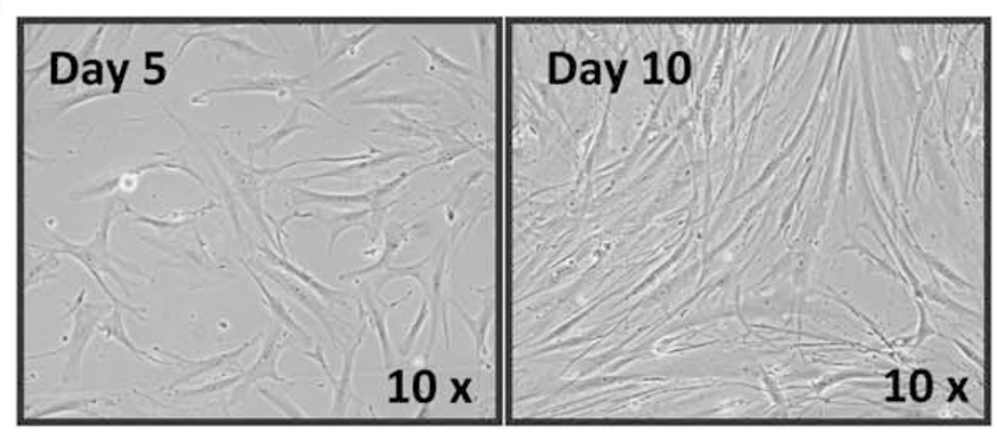

d $\mathrm{i}$

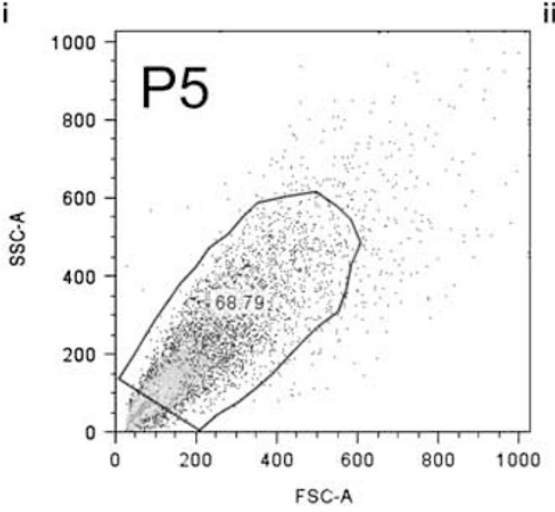

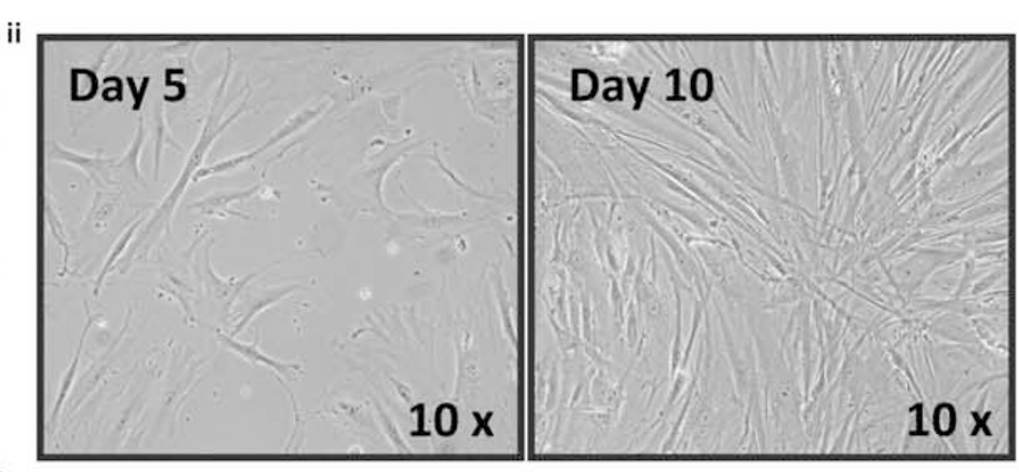


microscopy (Nikon Eclipse TE-2000U; Nikon Instruments Europe; Amstelveen, Netherlands).

\section{Adipogenic, Osteogenic and Chondrogenic Differentiation}

The potential of pHLP cells to differentiate into adipogenic, osteogenic and chondrogenic lineages were assayed at approximately day 10 on reaching confluency. Cells were harvested by accutase treatment and seeded in six-well culture plates at a cell density of $2.5 \times 10^{4} \mathrm{cells} / \mathrm{cm}^{2}$ in standard growth medium until $80 \%$ confluency. Cells were then stimulated under appropriate inducible conditions. Unstimulated cells were used as controls. For adipogenic differentiation, cells were stimulated using adipogenic differentiation media (Hyclone; Thermo Fisher Scientific, Erembodegem-Aalst, Belgium, Europe) supplemented with stem cell growth serum (Hyclone), according to manufacturer's instructions. Cells were maintained in adipogenic media for 3 weeks with media replenishment at every 3 days. Cells were washed thrice in PBS, washed twice in distilled water and covered with $0.3 \%$ oil red O Solution (Sigma) in isopropanol. After a 40-min incubation at room temperature, cells were washed thrice using distilled water. Similarly for osteogenic differentiation, cells were stimulated using osteogenic differentiation media (Hyclone) supplemented with stem cell growth serum (Hyclone), as per manufacturer's instructions. Cells were maintained in osteogenic media for 3 weeks with media replenishment at every 3 days. Cells were washed twice using 0.2-M Tris buffer, $\mathrm{pH}$ 8.3. Alkaline phosphatase activity was detected by the addition of BCIP/ NTP liquid substrate system (Sigma Aldrich) and incubation for $15 \mathrm{~min}$ at room temperature. Cells were washed twice in water and analyzed by phase-contrast inverted microscopy (Nikon Eclipse TE-2000U). For chondrogenic differentiation, a pellet culture of $1 \times 10^{6}$ cells in a conical tube was incubated with chondrogenic differentiation media containing stem cell growth supplements (Hyclone; Thermo Scientific), according to manufacturer's instructions. After 3 weeks of incubation, pellet was fixed in $4 \%$ formaldehyde (Dr Grogg Chemie AG, Stettlen-Deisswil, Switzerland) for $24 \mathrm{~h}$, dehydrated and infiltrated with paraffin using Citadel 2000 tissue processor (Histocom AG, Zug, Switzerland) before embedding. Sections of size $6 \mu \mathrm{m}$ were cut, placed onto superfrost slides (Thermo Scientific) and stained using Toluidine Blue O (Sigma Aldrich; Europe), according to the manufacturer's instructions. Stained sections were analyzed using a Leica
DMRB-transmitted light microscope equipped with Leica DC camera.

\section{RESULTS \\ Morphology and Growth of pHLP Cells}

Intraoperative surgical lung parenchyma samples were collected from 43 patients undergoing lung resection. Samples were taken from distal lung and were absent of any macroscopic signs of pneumonia, forms of infection or other parenchymatous disorders. Samples contained approximately 3.0-5.0 $\times 10^{6}$ cells $/ \mathrm{cm}^{3}$ of lung parenchyma tissue. Of the total number of cells plated onto tissue culture flasks, approximately $5 \%$ showed adherence to tissue culture flasks. At 24-h post cell seeding, the presence of thin fibroblast-like cells was apparent. Cells formed 1-2 individual colonies per $\mathrm{cm}^{2}$, which coalesced and grew to confluency at approximately day 10 in culture. The pHLP cell cultures had a predominant stromal-like morphology with some heterogeneity in cell size, shape and granularity. Sample to sample variability was evident and had some effect on cell morphology and growth of cell cultures. Forward and side scatter flow cytometric analysis did not show distinct subpopulations, and the morphological heterogeneity was considerably diminished by days 10-12. Figure 1 depicts representative flow cytometry profiles at passages $0,1,3,5$ and phasecontrast light microscopy images taken from corresponding samples at days 5 and 10 in culture. To rule out a possible source of cells from blood, we performed the same isolation and culture procedure with human blood and found neither cell attachment nor growth (data not shown).

\section{Immunophenotyping of pHLP Cells}

Confluent pHLP cell cultures were assessed by immunocytochemistry and/or harvested for flow cytometry analysis. Results from a representative culture are shown in Figure 2.

Primary pHLP cells expressed CD9 (cell motility marker), a stromal cell marker required for the regulation of pluripotency and differentiation of hematopoietic stem cells. In addition, we found that the pHLP cells expressed a number of cell surface antigens identified with mesenchymal stem cells. These included: CD73 (SH3), CD105 (SH2 or endoglin), CD166 (leukocyte cell adhesion molecule) and CD90 (Thy-1). Cells expressed low levels of STRO-1, a marker identified with some stromal cell precursors. We confirmed that cells do not express: CD34, a hematopoietic stem cell antigen; CD45, a leukocyte marker; CXCR4, a hematopoietic stem cell antigen; and CD31, an endothelial cell marker. Finally, we confirmed the

Figure 2 Immunophenotypic analysis of primary human lung parenchyma cells before passaging. (a) Representative pHLP cell flow cytometry profile showing that cultures express established mesenchymal stem cells markers: CD9, CD166, CD73, CD105 and STRO1. (b) Representative pHLP cell flow cytometry profile showing that cultures are devoid of hematopoietic markers CXCR4, CD45 and CD34, and endothelial cell marker, CD31. (c) Intracellular flow cytometry analysis illustrates that pHLP cell cultures do not express Clara cell marker, CCSP, and type I alveolar cell marker, AQ5. Cells do express some expression of the type II alveolar cell maker, SPC. Positive expressions of cells are depicted by the solid green histograms (a measure of cell reactivity to respective monoclonal antibodies as detected by Alexa488-conjugated secondary antibody) in comparison with the appropriate isotype control represented by the dashed blue line histograms. 
a
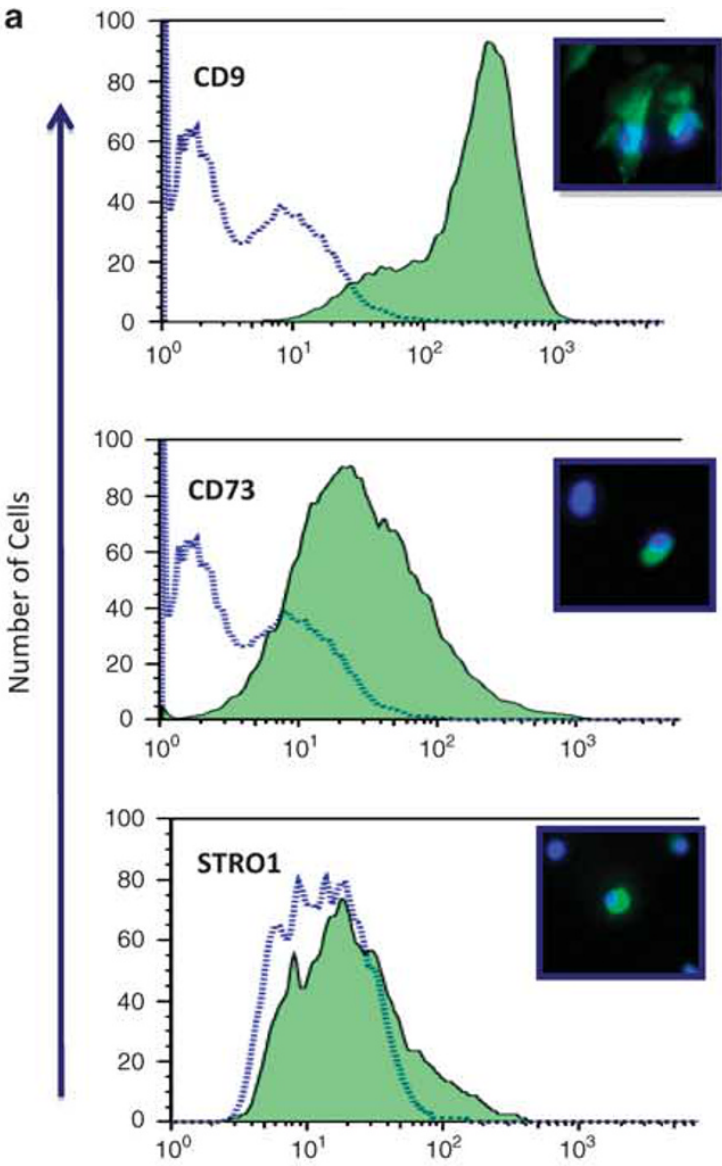
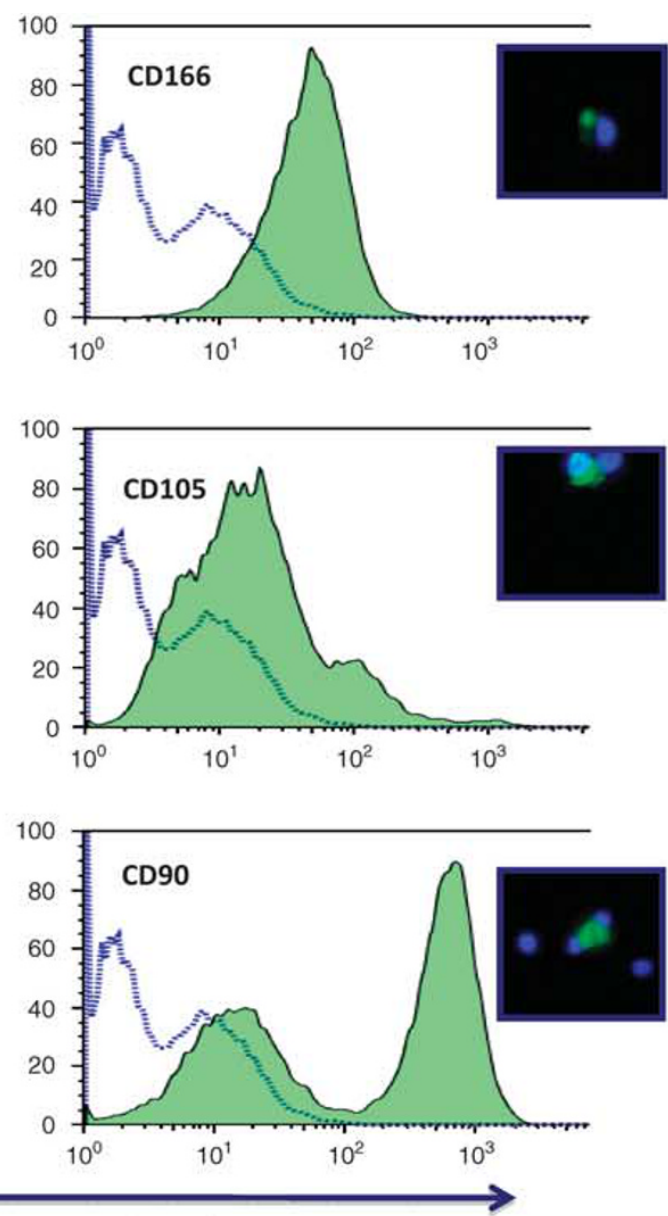

Alexa488 Fluorescence Intensity
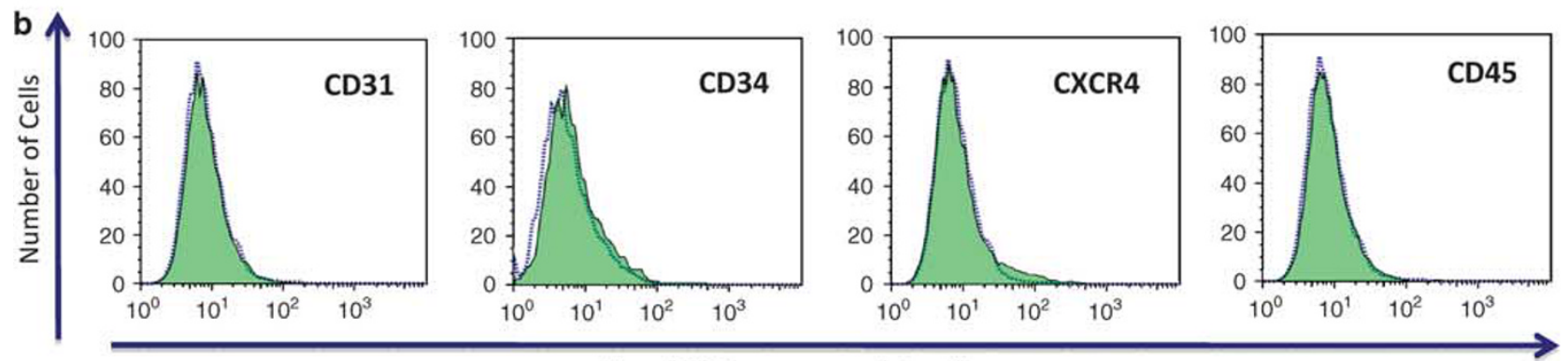

Alexa488 Fluorescence Intensity
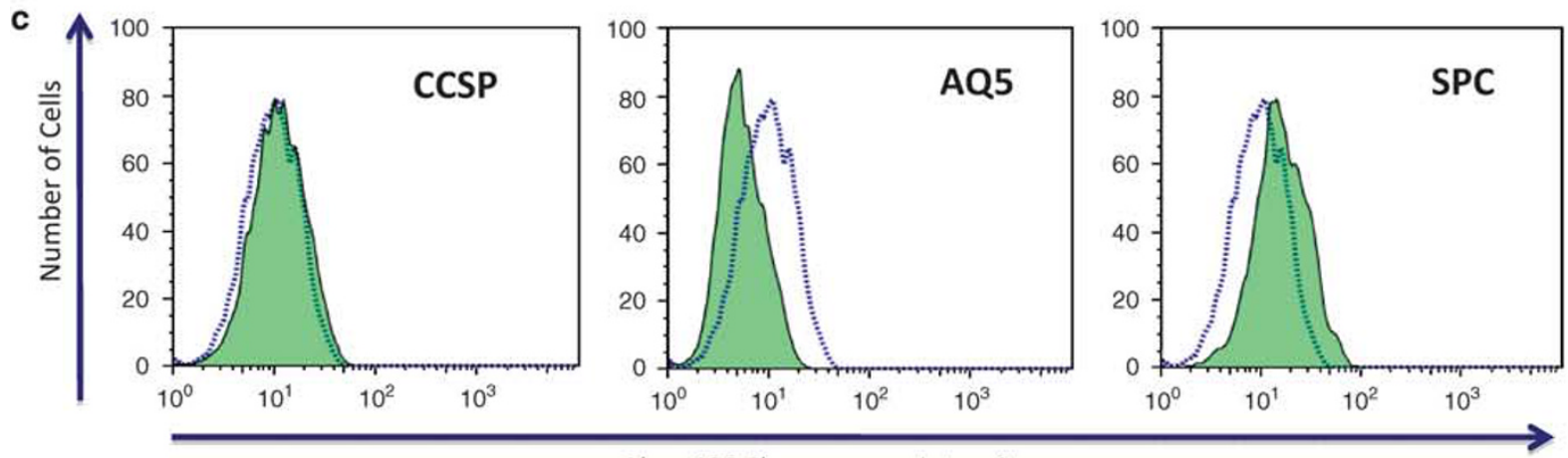

Alexa488 Fluorescence Intensity 
negative expression of CD11b, CD79alpha, CD19 and HLADR molecules (data not shown). Together, this pattern of antigen expression is consistent with a MSC phenotype.
To further characterize the pHLP cells, we examined whether the primary cultures contained differentiated airway epithelial cells. The presence of alveolar epithelial types I a

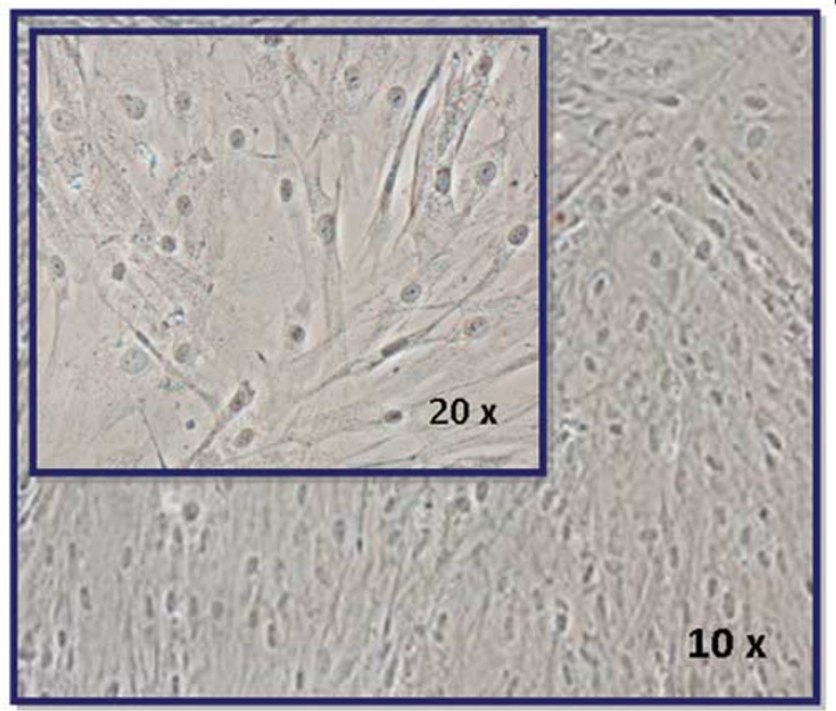

c

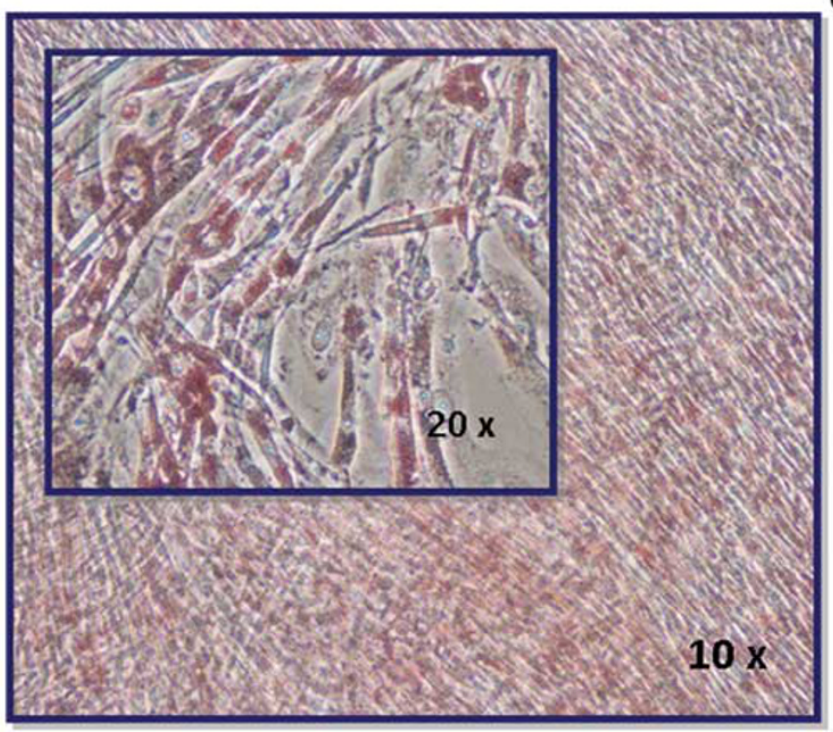

b

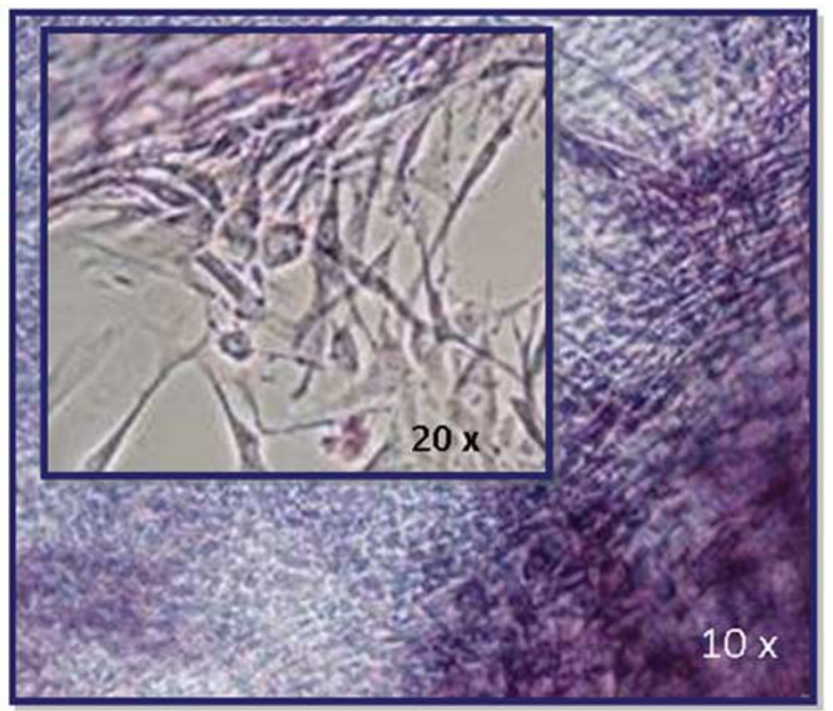

d

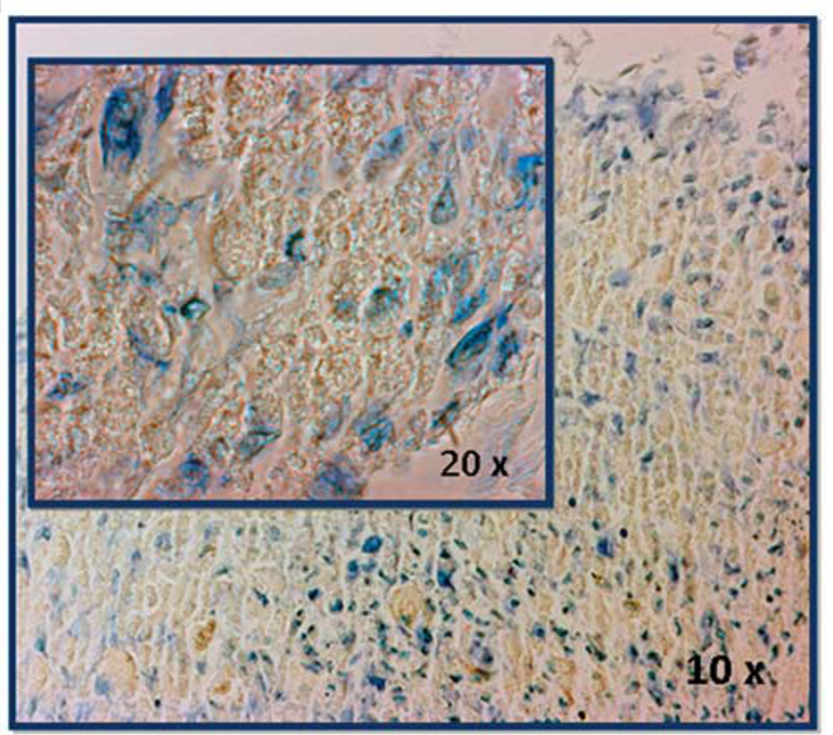

Figure 3 Adipogenic, osteogenic and chondrogenic differentiation of pHLP cell cultures. Cells were cultured in control (a), osteogenic-inducing media (b), adipogenic-inducing media (c) or chondrogenic-inducing media (d) for 3 weeks. Osteogenic differentiation was assessed by BCIP/NTP staining for alkaline phosphatase activity represented by the blue staining. Accumulation of lipid droplets within the cells was revealed by staining with oil red $O$ and viewed under an inverted phase-contrast microscopy. Toluidine blue staining was used for the detection of chondroitin sulfate, and stained sections were analyzed using a Leica DMRB-transmitted light microscope equipped with Leica DC camera.

Figure 4 Differentiation of pHLP cell cultures into lung airway and alveolar cells at P0. Expression of type II alveolar cell marker, SPC, Clara cell marker, CCSP, and type I alveolar cell marker, AQ5, was detected using both flow cytometry analysis as well as immunocytochemistry. (a) Expression of SPC, CCSP and AQ5 after 1 day in SAGM differentiation conditions. Positive expression of cells, as detected by flow cytometry, is depicted by the solid green histograms in comparison with the appropriate isotype controls represented by the dashed blue line histograms (top panel). For the immunocytochemistry studies, cells were stained with anti-human SPC, CCSP and AQ5 (green), and propidium iodide nuclear staining (red) (bottom panel). (b) Expression of SPC, CCSP and AQ5 after 7 days in SAGM differentiation conditions. Positive expression of cells, as detected by flow cytometry, is depicted by the solid green histograms in comparison with the appropriate isotype controls represented by the dashed blue line histograms (top panel). For the immunocytochemistry studies, cells were stained with anti-human SPC, CCSP and AQ5 (green), and propidium iodide nuclear staining (red) (bottom panel). Images were acquired using inverted fluorescence microscopy and are at $\times 10$ magnification (inserts at $\times 40$ magnification). 

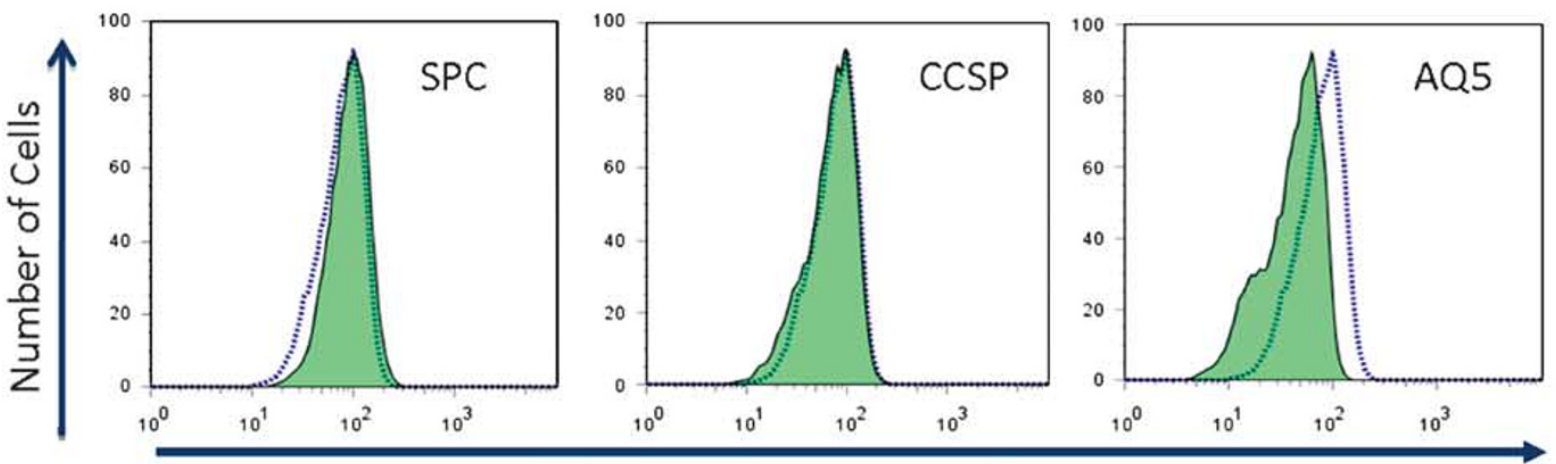

Alexa488 Fluorescence Intensity
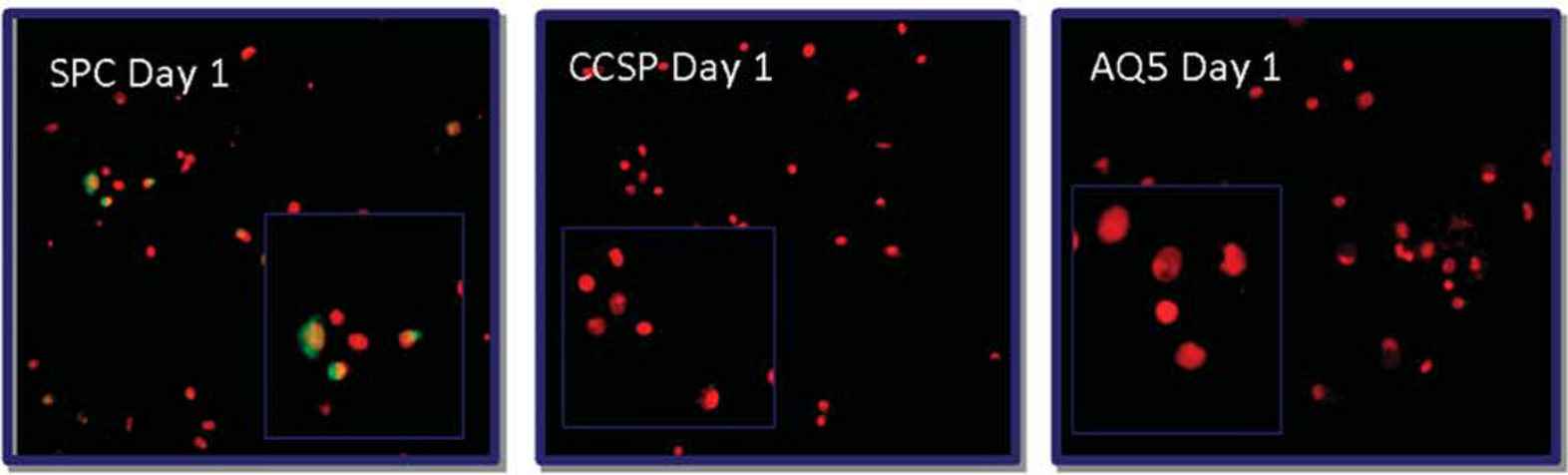

b

Day 7 in SAGM Differentiation Media
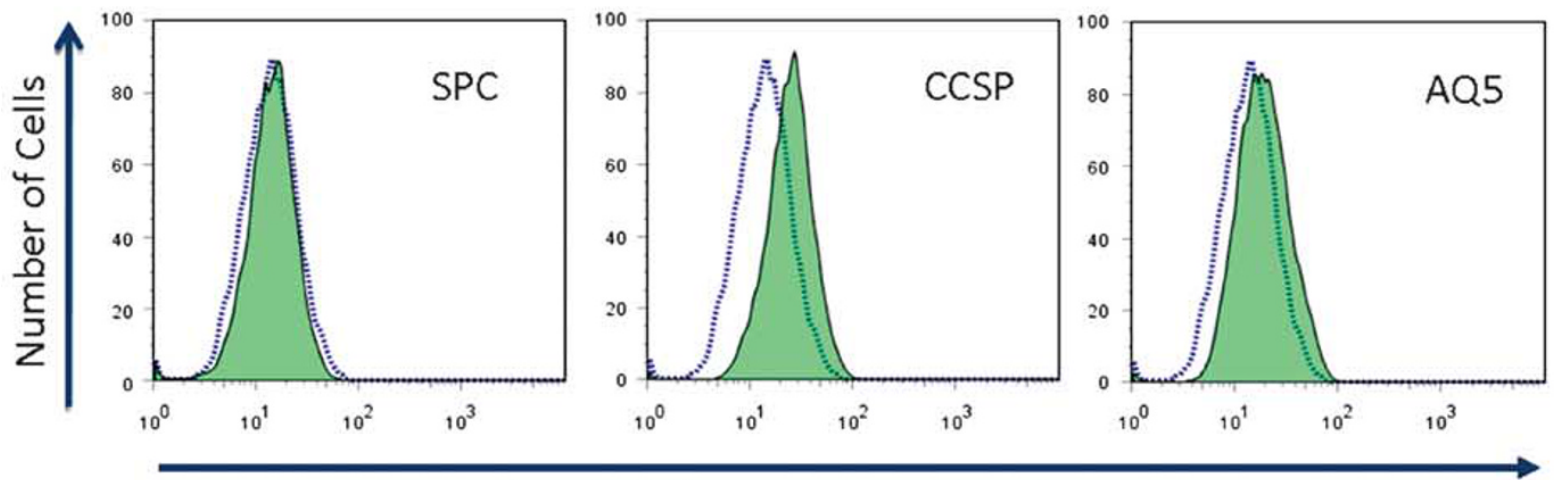

Alexa488 Fluorescence Intensity
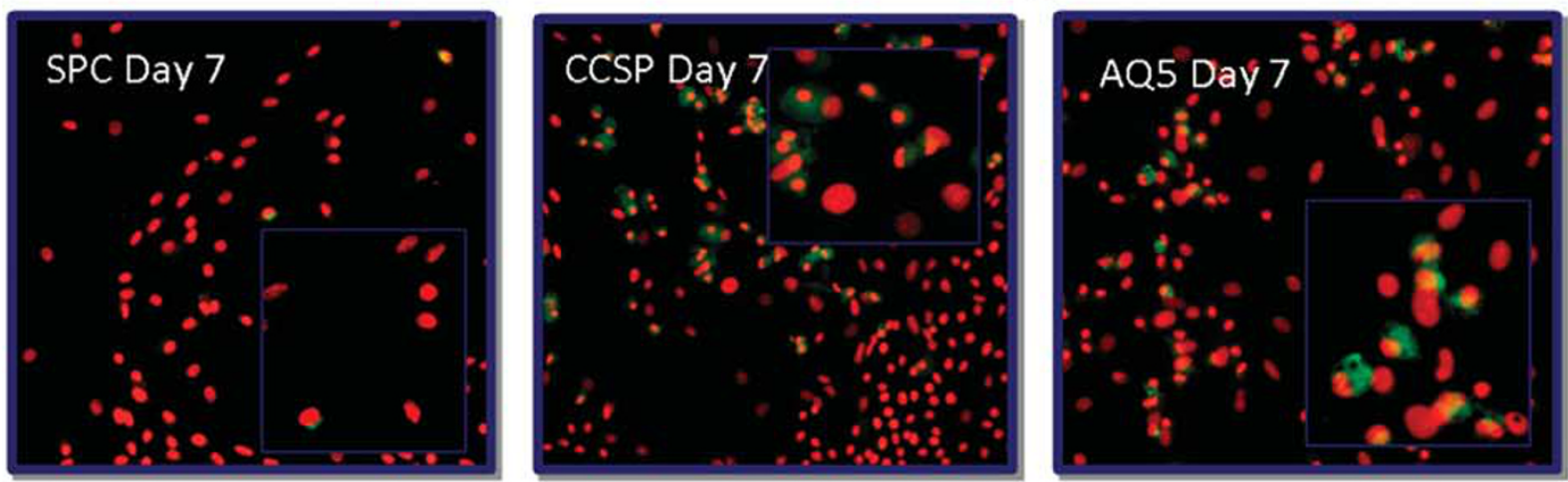
and II was assessed using intracellular flow cytometry analysis for AQ5 and SPC, respectively. Similarly, we looked for the existence of Clara cells by tracking intracellular CCSP expression. Figure $2 \mathrm{~b}$ depicts the intracellular flow cytometry profile of a representative pHLP cell culture. pHLP cells were negative for type I alveolar cell marker, AQ5. In contrast, the expression of low levels of the type II alveolar cell marker, SPC, was present. Similarly, cultures contained no Clara cells, as indicated by the negligible expression of CCSP.

\section{pHLP Cells Show Osteogenic, Adipogenic and Chondrogenic Differentiation}

Given the observed MSC cell surface antigen profile in the pHLP cell cultures, we inspected the potential of the cells to differentiate along mesodermal lineages. Specifically, we examined differentiation along the osteogenic, adipogenic and chondrogenic lineages. To induce osteogenic differentiation, cells were cultured on type I collagen-coated dishes in differentiation medium for 3 weeks. Alkaline phosphatase activity, an indicator of osteogenic differentiation, was determined using BCIP/NTP liquid substrate system and represented by the blue staining. Results from a representative differentiation assay are illustrated in Figure $3 \mathrm{~b}$ and show differentiation along the osteogenic lineage in the presence of inducible culture conditions. To examine the ability of the pHLP cells to differentiate along the adipogenic lineage, cells were placed in adipogenic-inducing media. After 3 weeks, cultures were stained with oil Red $\mathrm{O}$ to detect lipid production, as evidenced by the red spots denoting the formation of lipids (Figure 3c). A marked adipogenesis was inducted in the pHLP cells under inducible culture conditions. In the absence of adipogenic and osteogenicinducible factors, no differentiation was observed (Figure 3a). For chondrogenic differentiation, pHLP cells were pelleted and maintained in chondrogenic-inducible media for 3 weeks. Toluidine blue staining was used for the detection of chondroitin sulfate, which was clearly detected in the cell cultures. These results further confirm the multipotent MSC identity of the human lung parenchyma cells.

\section{Differentiation of pHLP Cells into Airway Epithelial Cells}

To explore as to the possible differentiation capabilities of the pHLP cells into mature cells of the lung, we performed the small airway growth media (SAGM) differentiation assay. Confluent pHLP cell cultures were harvested by accutase treatment, replated and maintained in SAGM for 7 days. The cells were harvested for flow cytometry analysis or fixed for immunocytochemistry for type I alveolar cells, type II alveolar cells and for Clara cells. As controls, the pHLP cell cultures were grown in the regular pHLP cell MCDB growth media and in the high-serum containing media (DMEM $+10 \%$ FBS $+1 \%$ P/S $)$. Differentiation experiments were conducted on four different pHLP cell culture samples at passage 0 . Results from a representative sample are illu- strated in Figure 4. Flow cytometry after $24 \mathrm{~h}$ in SAGM media, showed no presence of type I alveolar cells or Clara cells. In contrast, there was a low expression of SPC, indicating the presence of type II alveolar cells in the cultures (Figure 4a; top panel). These results were confirmed by immunocytochemistry (Figure 4b; bottom panel). Analogous analysis after 7 days, however, elucidated cells positive for the expression of AQ5 as well as CCSP with a slight reduction in SPC-expressing cells (Figure 4b; top panel). Note that no expression of AQ5 or CCSP was observed in cells cultured in both control conditions (data not shown).

\section{pHLP Cells Maintain MSC Characteristics and Their} Ability to Give Rise to AQ5 + Cells in Long-term Culture pHLP cell cultures were expanded in culture by passaging when approximately $85 \%$ confluent and subsequently replated at a low cell density $\left(1000\right.$ cells $\left./ \mathrm{cm}^{2}\right)$. Cells were cultured up to passage 6 (P6) with phenotype assessment at each passage. We found that the cells maintained their P0 MSC phenotype with the exception of STRO-1, which is no longer present at $\mathrm{P} 1$. The remaining markers, including CD166, CD73, CD90, CD9 and CD105, were strongly expressed at $\mathrm{P} 1$ and were sustained at the later passages. Figure $5 \mathrm{a}$, illustrates representative flow cytometry profiles showing the consistent MSC signature phenotype at P1 and P5, respectively. We assessed the potential of the pHLP cells at subsequent passages to differentiate along the osteogenic, adipogenic and chondrogenic lineages. As can be appreciated in Figure 5b, under inducible conditions, pHLP cell cultures illustrate alkaline phosphatase activity, Toluidine blue staining for chondrocytes and significant lipid production at P1 (top panel) and P5 (bottom panel), respectively.

Finally we explored whether the cells were able to differentiate into type I alveolar epithelial cells using quantitative real-time PCR to examine the expression of AQ5, CCSP and SPC (Figure 6). Figure 6a shows that in agreement with our flow cytometry and immunofluorescence staining results, there are low but detectable levels of SPC in the absence of SAGM media, with no significant change in the expression over the 7-day culture in SAGM. Conversely, at P0, we observe the expression of both AQ5 and CCSP even in the absence of SAGM differentiation media, seemingly undetectable at the cellular level. In the presence of SAGM, there is a rise in AQ5 mRNA levels and in CCSP (Figure 6a). Analysis at passages P1 (Figure 6b) and P5 (Figure 6c) depicts the same observed trend with respect to AQ5, showing increasing levels of AQ5 over the 10-day culture period in SAGM differentiating conditions. In contrast, we did not observe any expression of CCSP or SPC at the later passages.

\section{Presence of SP Cells in the pHLP cell Cultures}

Recent studies have implicated SP cells as a likely source for the MSCs observed in murine adult lung. On the basis of these reports, ${ }^{42,44}$ we speculated that there may likely be a rare subset of SP cells in the pHLP cell cultures, which are 

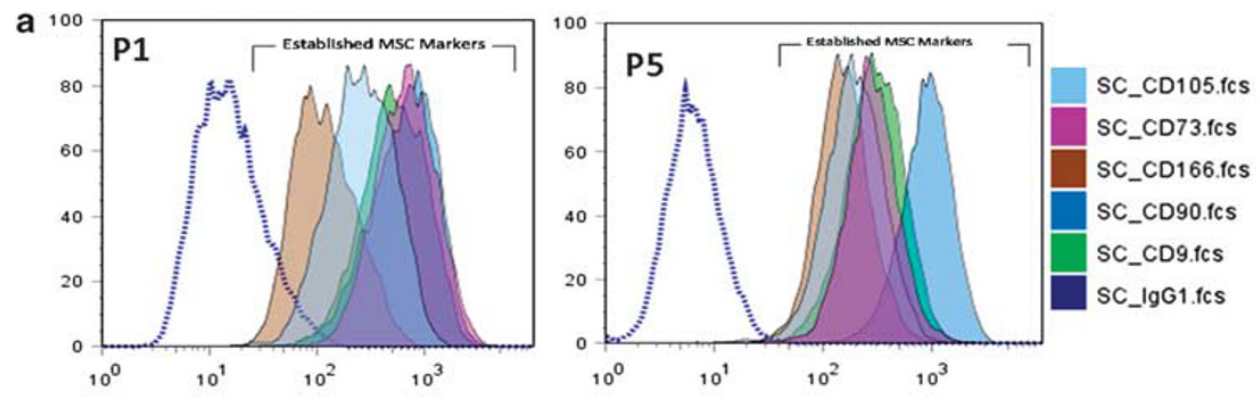

b

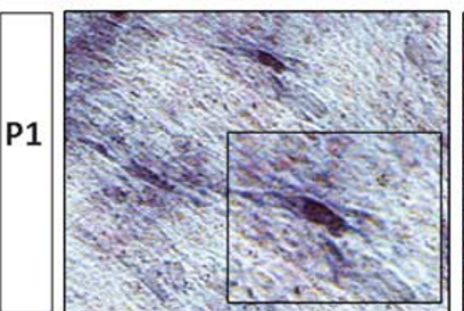

Adipogenic

Chondrogenic
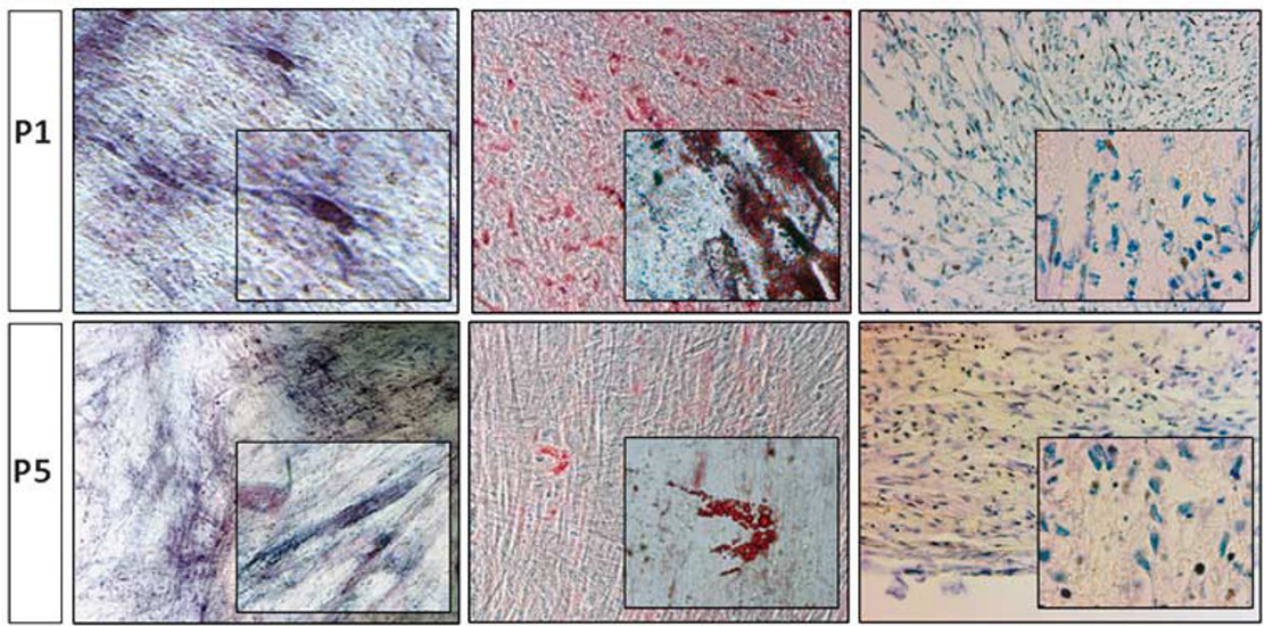

Figure 5 Evaluation of pHLP cell phenotype and differentiation capacity in long-term culture. (a) Flow cytometry immunophenotypic analysis of established MSC markers, as depicted by the colored solid histograms over the negative isotype control (dotted histogram). (b) Phase-contrast light microscopy images $(\times 10$; insert at $\times 40)$ depicting differentiation along the osteogenic, adipogenic and chondrogenic lineages.

potentially the source of the mesenchymal stem cell-like population. SP cells are characterized by their unique ability to efflux the vital dye, Hoechst 33342, through the action of the $\mathrm{ABC}$ transporter multidrug-resistant protein, ABCG2. ${ }^{45}$ The NCI-H460 lung cancer cell line was used as a positive control and this clearly revealed the incidence of SP cells accounting for approximately $1.8 \%$ of the total $\mathrm{H} 460$ cell population. As expected, cell treatment with $\mathrm{ABC}$ transporter inhibitor, verapamil, dramatically decreased the percentage of SP cells. Interestingly, we found a rare $(0.02-0.05 \%)$ subset of the primary cultures characteristic of the SP phenotype. Similar to the NCI-H460 cell line, treatment with verapamil abrogated this population (Figure 7a) confirming that the extrusion of the toxins was a function of multidrug-resistant proteins within the cells. To ensure the occurrence of the ABCG2 multidrugresistant protein, we inspected its transcript in the cell culture samples. We tested four different pHLP cell cultures derived from clinical samples and found the expression of the ABCG2 (200 bp) in all four. The lung cancer cell lines NCI-H460 and NCI-A549 were used as positive controls (Figure 7b).

\section{DISCUSSION}

In the present study, we have identified a population of mesenchymal stromal cells in human lung parenchyma capable of differentiating into AQ5-expressing cells.
MSCs have now been isolated from a wide variety of tissues, ${ }^{26-31}$ including adult mouse lungs, ${ }^{42,44}$ from bronchial aspirates of both neonates ${ }^{38}$ and from lung transplant patients. ${ }^{39}$ Although MSCs isolated from each of these sources generally express comparable cell surface antigens, differences in levels of expression and lineage differentiation tendencies have been observed. Sabatini et al, ${ }^{40}$ illustrated that cells derived from bronchial biopsies expressed CD73, CD105, CD166, no expression of CD90 and low levels of STRO-1. In contrast, Hennrick et al, ${ }^{38}$ reported that cells derived from tracheal aspirates of neonates expressed high levels of STRO-1 and CD90 in addition to CD73, CD105 and CD166. Both studies showed that cells were devoid of hematopoietic markers and had the ability to differentiate along osteogenic and adipogenic lineages. Here, we illustrate that cells isolated from human lung parenchyma also show MSC properties. In agreement with previous reports, our cells lack hematopoietic antigens (CD34, CD45 and CXCR4) and endothelial cells (CD31). Our pHLP cells express CD90, CD73, CD105 and with low levels of STRO-1.

In addition to the established MSC surface antigens previously reported in the lung, we find that our pHLP cells are highly positive for CD9. Otherwise known as the motilityrelated protein-1, CD9 is a member of the tetraspanin family of proteins implicated in a number of different cellular 

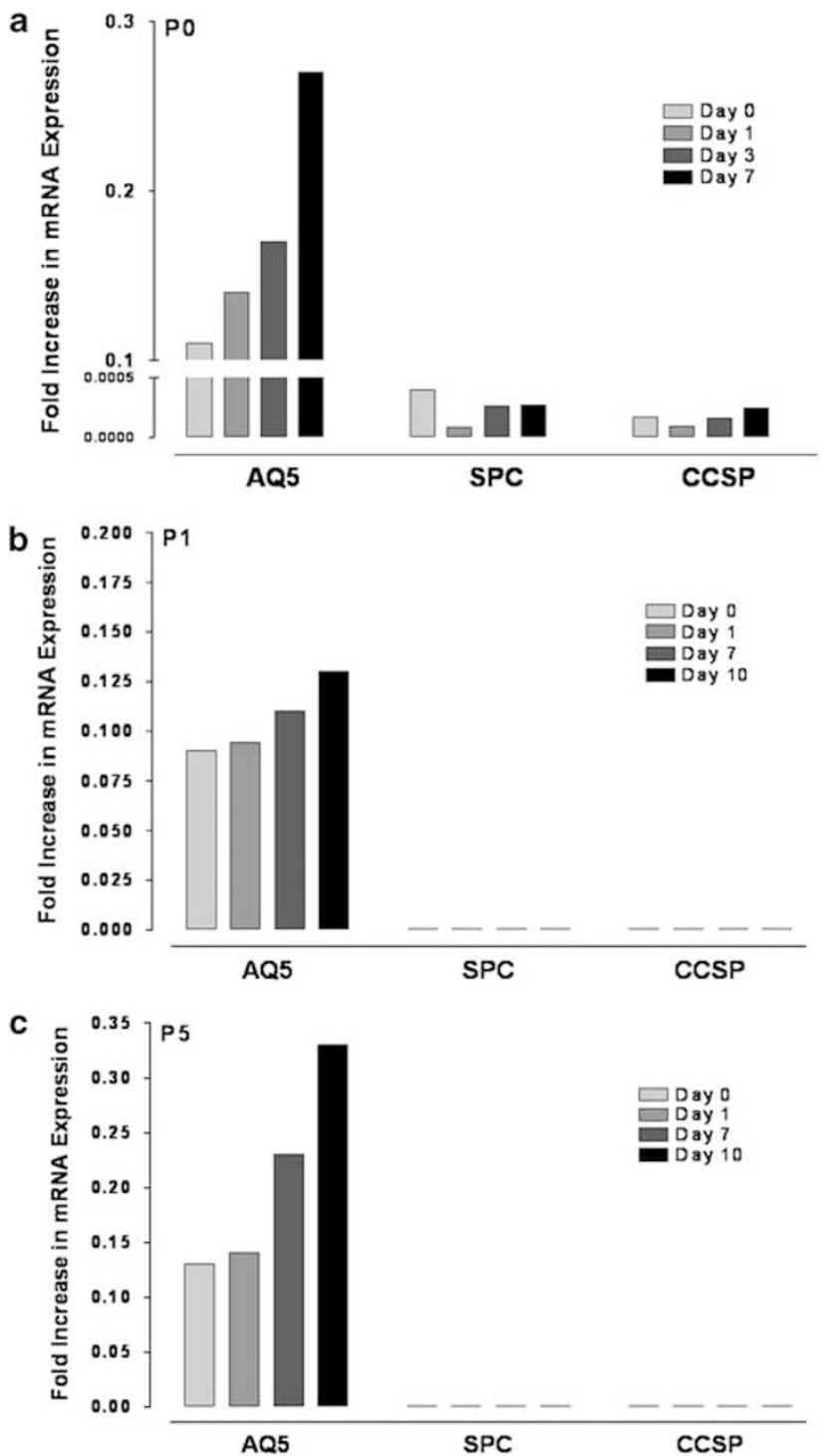

Figure 6 pHLP cell culture mRNA expression of Aquaporin 5 (AQ5), Surfactant protein C (SPC) and Clara cell secretory protein (CCSP) in small airway growth media (SAGM) differentiation-inducing culture conditions. Data are expressed as fold change in mRNA expression of AQ5, SPC and CCSP, as detected in representative PHLP cell cultures, relative to snap-frozen normal human lung parenchyma tissue at (a) P0; (b) P1; and (c) P5.

functions, including adhesion, migration, differentiation and signal transduction. ${ }^{46-50}$ Interestingly, varying degrees of CD9 have been found in human MSCs and have an essential role for stromal cell control of hematopoiesis. ${ }^{51}$ More recently, CD9 has been shown to be important for human adipose-derived MSC adhesion and proliferation resulting in enhanced engraftment and differentiation in a hindlimb ischemia model. ${ }^{46}$ Though the exact mechanism remains elusive, several hypotheses have been postulated, including complex formation with other proliferation signaling molecules, ${ }^{46,52}$ integrin association and NF- $\kappa \mathrm{B}$ activity. In the lung, CD9 has been detected in the alveolar wall, alveolar epithelial cells and bronchiolar epithelium. ${ }^{53}$ In addition, its expression has been found to be markedly reduced in lung cancer. ${ }^{54,55}$ Taken together, there seems to be an important role for $\mathrm{CD} 9$ in the lung. Here, we illustrate for the first time, that MSCs derived from normal human lung parenchyma express CD9. The specific role of CD9 in the proliferative and differentiation capabilities of these cells remains to be determined.

MSCs are currently characterized by their adherence to a plastic surface, a panel of cell surface markers and their ability to differentiate along mesodermal lineages. We investigated whether the pHLP cell cultures can give rise to cells of the adipogenic, osteogenic and chondrogenic lineages. After transfer to specific induction media, pHLP cells assumed distinct differentiated cell fates and illustrated the ability to differentiate along all three lineages. For the pHLP cells to be considered as a potential source for cell-based therapies to the lung, they must have the capacity to give rise to alveolar epithelial cells. We tested the capacity of the pHLP cell cultures (before passaging) to differentiate into airway epithelial cells by culturing the cells in SAGM, a previously established lung differentiation culture media. ${ }^{5-58}$ Our data indicate that the pHLP cell cultures have the ability to acquire markers specific for Clara cells of the airway as well as type I alveolar epithelial cells after only 1 week in SAGMinduced differentiation conditions. Immunophenotypic analysis depicted the appearance of AQ5-positive cells in cultures after 7 days in SAGM differentiation media. In addition, we observed a number of CCSP-positive cells showing differentiation into Clara cells.

These data suggest that the pHLP cell cultures have the capacity to acquire mature lung phenotypic markers under appropriate differentiation conditions.

According to the conventional paradigm, type I alveolar epithelial cells are terminally differentiated cells, incapable of dividing and type II cells are the only progenitors cells of the alveolar epithelium. It is important to note, however, that new concepts in adult stem cell biology now suggest that there is more complexity than that portrayed by conventional beliefs. First, several studies have now illustrated that it is possible for type I cells to re-enter a cell cycle phase, leading to proliferation and differentiation into type II cells, ${ }^{59-62}$ hence challenging the 'terminally differentiated' notion. Second, rapidly accumulating new evidence suggests other potential progenitor pools for type I epithelial cells. Among these are studies on lung embryos, which have illustrated coexpression of markers specific for both types I and II cells. ${ }^{63,64}$ Resident putative 'stem cell' populations, such as the bronchi-alveolar stem cells, ${ }^{17}$ have now been identified and have been shown to regenerate damaged epithelium. Importantly, there have been numerous studies implicating bone marrow-derived cells as an exogenous progenitor cell pool contributing to type I cell regeneration. ${ }^{6,20,58,65,66}$ In support of this new paradigm are data from human 
a

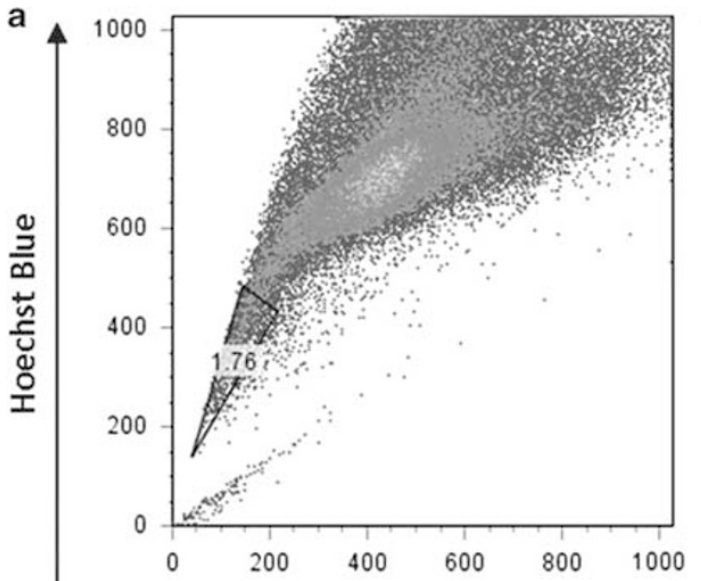

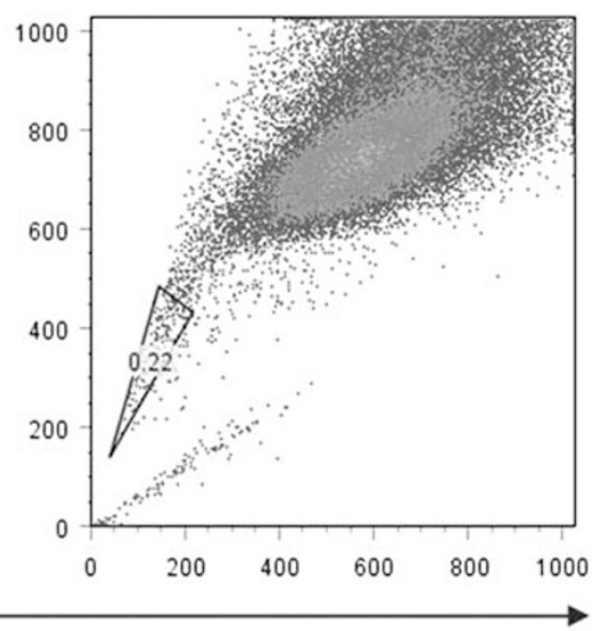

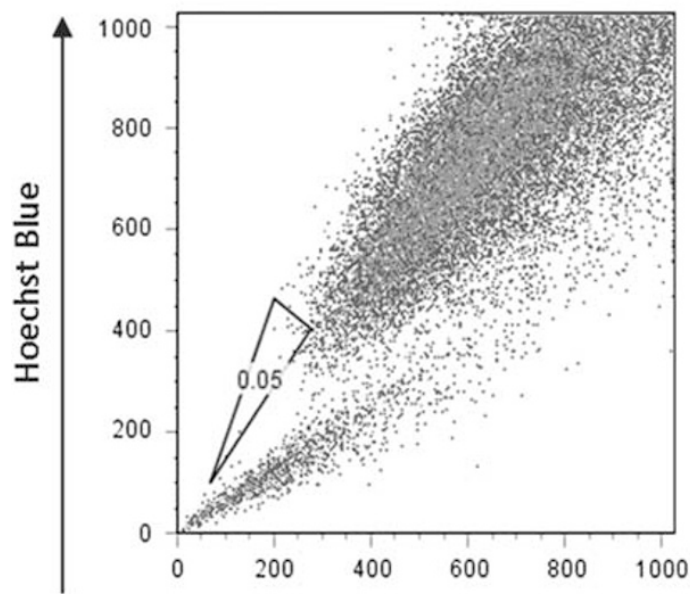

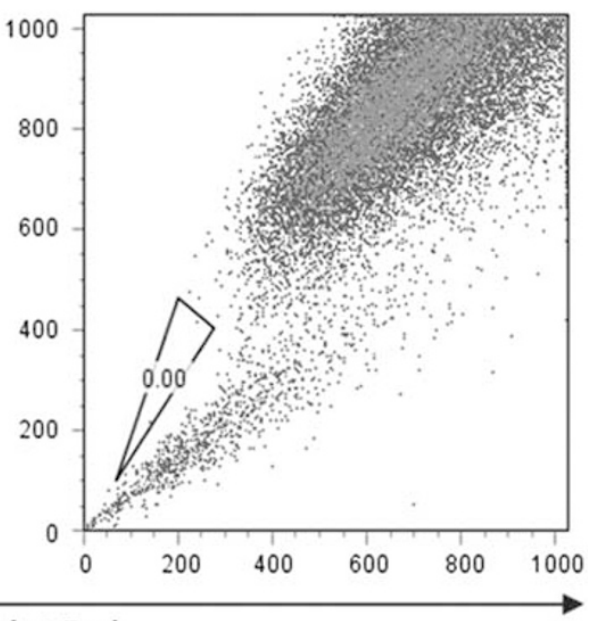

Hoechst Red

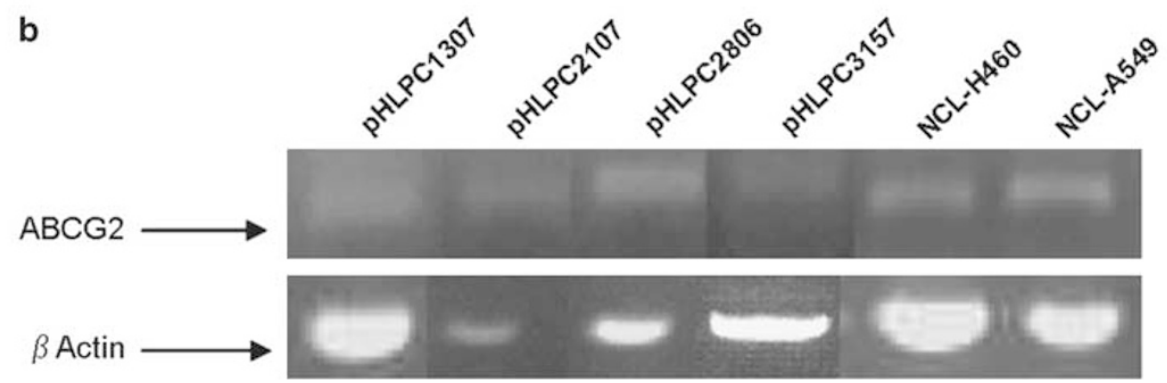

Figure 7 Presence of 'side population' cells in primary human lung parenchyma cell cultures. (a) Density dot plot showing SP cells in the NCl-H460 lung cancer cell line in the absence (top panel-left) and presence (top panel-right) of verapamil. pHLP cell cultures illustrate a small percentage of cells with the side population phenotype (bottom panel-left). This population is eliminated in the presence of the ABCG2 inhibitor, verapamil (bottom panel-right). (b) ABCG2 (200 bp) transcript expression in representative pHLP cell cultures.

transplant studies, which show that human lung biopsies taken from bone marrow transplant recipient patients show chimerism for epithelial cells. ${ }^{67}$ In addition, other studies of recipients of sex-mismatched marrow transplants have found donor-derived epithelial cells in the lungs. ${ }^{68}$

Our gene expression analysis at $\mathrm{P} 0$ shows a rise in AQ5 + expression and low but detectable levels of SPC and CCSP.
It is possible that the SPC + and, potentially, the CCSP + cells serve as an endogenous progenitor pool for the AQ5expressing cells. In contrast, on passaging (P1-P5), the expression of SPC and CCSP is lost. We hypothesize that this can be attributed to (1) unsuitable culture conditions and/or media resulting in cell death; or (2) an exhaustion of both of these resident progenitor pools directly resulting from 
differentiation into AQ5. These data suggest two possible explanations: First, that the increase in AQ5 + cells results from existing SPC + and/or CCSP + epithelial progenitor cells which then expand in culture and second, that that the AQ5 + cells are potentially derived from a committed progenitor in the pHLP cells population. In the second case, we hypothesize that there are committed progenitors in the human lung parenchyma MSC cultures that, when placed in the right microenvironment, give rise to AQ5 + cells. In support of this idea are recent studies depicting committed airway epithelial progenitor pools in umbilical cord blood and bone marrowderived MSCs. Sueblinvong et al, show that in air-liquid interface culture, human cord blood-derived MSCs express phenotypic markers of airway epithelium and when transplanted in vivo participate in airway remodeling. ${ }^{58}$ In another study, Wong et al, ${ }^{66}$ identified a CCSP + bone marrow-derived epithelial-like population, which they believe to be a committed epithelial progenitor sub-population in the bone marrow, capable of repopulating injured murine lung. Although we believe that the first case is likely and to a certain extent, fits with the conventional paradigm, we continue to see a rise in AQ5 expression despite a lack of SPC and CCSP. Hence, we can conclude that the contribution of a committed progenitor cell(s) from an endogenous or exogenous cell pool is partially involved. We are currently investigating as to the identity of the AQ5 + committed progenitors.

Isolated SP cells from adult murine lung have been recently identified as a potential source of the mesenchymal stem cells previously described in tracheal aspirates from neonates in respiratory distress and from adult bronchoalveolar lavage. ${ }^{42,44} \mathrm{SP}$ cells are a rare subset of primitive and undifferentiated cells with a unique ability to efflux the vital dye, Hoechst 33342. They can be detected using flow cytometry-based analysis, which depicts a lateral position on a 'blue/red' fluorescence cytometric dot plot as an 'off the side' subset of the main population. ${ }^{45} \mathrm{SP}$ cells have been identified in several adult stem cell populations showing stem cell properties, including contribution to diverse lineages. ${ }^{69-71}$ We sought to assess for the incidence of SP cells in our pHLP cell cultures. And in fact, we illustrate approximately $0.05 \%$ of cells in the pHLP cell cultures with the SP cytometric profiles. This result is in agreement with the SP percentages found in other stem and/or progenitor cell populations. The exclusion of Hoechst 33342 by SP cells is an active process involving ABCG2 and, therefore, treatment of cells with ABCG2 inhibitor, verapamil, attenuates SP cell numbers by blocking the ABCG2 pump. ${ }^{72-74}$ In support of the SP data, gene expression analysis indicated the presence of the ABCG2 transcript confirming the presence of the SP phenotype in the pHLP cells. We are currently isolating and analyzing single SP clones to determine whether, in fact, SP cells are the source of the human lung parenchyma MSCs.

The familiar local milieu and lung microenvironment make the resident progenitor cell population an ideal cell source for regenerative cell therapy to the lung. For clinical applicability, however, we not only need to define and stringently characterize putative endogenous stem/progenitor cells but also will require methodologies to successfully culture and expand these cells. Only then, will we begin to make progress toward paving the way for autologous lung stem/ progenitor cell therapy. In this report, we describe a population of MSC cells derived from human lung parenchyma illustrating differentiation potential. Especially of note is the differentiation of the pHLP cells into alveolar and airway epithelium. Although these cells have to be further examined and assessed for both engraftment and differentiation in vivo, we believe that we have enough evidence to warrant further investigation of these cells as a potential therapeutic population for the lung. The use of these pHLP cells would support a strategy in which enhancement of the physiological repair and regeneration mechanisms are provided by the lung's own endogenous progenitor cell population.

\section{DISCLOSURE/CONFLICT OF INTEREST}

The authors declare no conflict of interest.

1. UNOS. United Network for Organ Sharing. www.optn.org/latestData/ rptSrat.aspDate last updated: 25 January 2008. Date last accessed: 31 January 2008.

2. Stripp B, Shapiro S. Stem cells in lung disease, repair and the potential for therapeutic interventions. Am J Respir Cell Mol Biol 2006;34: 517-522.

3. Mimeault M, Hauke R, Batra SK. Stem cells: a revolution in therapeuticsrecent advances in stem cell biology and their therapeutic applications in regenerative medicine and cancer therapies. Clin Pharmacol Ther 2007;82:252-264.

4. Loebinger MR, Aguilar S, Janes SM. Therapeutic potential of stem cells in lung disease: progress and pitfalls. Clin Sci (Lond) 2008;114:99-108.

5. Burt R, Pearce W, Luo K, et al. Hematopoietic stem cell transplantation for cardiac and peripheral vascular disease. Bone Marrow Transplant 2003;32:S33-S35.

6. Krausse $D$, Theise $N$, Collector $M$, et al. Multi-organ, multi-lineage engraftment by a single bone marrow derived stem cell. Cell 2001;105:369-377.

7. Allers C, Sierratta W, Neubauer S, et al. Dynamic of distribution of human bone marrow derived mesenchymal stem cells after transplantation into adult unconditioned mice. Transplantation 2004;78:503-508.

8. Anjos-Afonso $F$, Siapati E, Bonnet D. In vivo contribution of murine mesenchymal stem cells into multiple cell-types under minimal damage conditions. J Cell Sci 2004;117:5655-5664.

9. Albera C, Polak J, Janes $S$, et al. Repopulation of human pulmonary epithelium by bone marrow cells: a potential means to promote repair. Tissue Eng 2005;11:1115-1121.

10. Fine A. Marrow cells as progenitors of lung tissue. Blood Cells Mol Dis 2004;32:95-96.

11. Ohnishi S, Yanagawa B, Tanaka K, et al. Transplantation of mesenchymal stem cells attenuates myocardial injury and dysfunction in a rat model of acute myocarditis. J Mol Cell Cardio 2007;42:88.

12. Rojas $M, X u J$, Woods $C$, et al. Bone marrow-derived mesenchymal stem cells in repair of the injured lung. Am J Respir Cell Mol Biol 2005;33:145-152.

13. Engelhardt JF, Schlossberg H, Yankaskas JR, et al. Progenitor cells of the adult human airway involved in submucosal gland development. Development 1995;121:2031-2046.

14. Hong $K$, Reynolds $S$, Giangreco A, et al. Clara cell secretory protein expressing cells of the airway neuroepithelial body microenvironment include a label retaining subset and are critical for epithelial renewal after progenitor cell depletion. Am J Respir Cell Mol Biol 2001;24: $671-681$. 
15. Liu X, Driskell RR, Engelhardt JF. Stem cells in the lung. Methods Enzymol 2006;419:285-321.

16. Giangreco A, Reynolds SD, Stripp BR. Terminal bronchioles harbor a unique airway stem cell population that localizes to the bronchoalveolar duct junction. Am J Pathol 2002;161:173-182.

17. Kim C, Jackson E, Woolfenden A, et al. Identification of bronchioalveolar stem cells in normal lung and lung cancer. Cell 2005;121:823-835.

18. Majka $S$, Beutz $M$, Hagen $M$, et al. Identification of novel resident pulmonary stem cells form and function of the lung side population. Stem Cells 2005;23:1073-1081.

19. Huang D, Hung $Y, K o B$, et al. Highly efficient cellular labeling of mesoporous nanoparticles in human mesenchymal stem cells: implication for stem cell tracking. FASEB J 2003;5:1-24.

20. Kotton D, Ma B, Cardoso W, et al. Bone marrow-derived cells as progenitors of lung alveolar epithelium. Development 2001;128: 5181-5188.

21. Kotton $D$, Fabian $A$, Mulligan R. Failure of bone marrow to reconstitute lung epithelium. Am J Respir Cell Mol Biol 2005;33:328-334.

22. Ortiz L, Gambelli F, McBride C, et al. Mesenchymal stem cell engraftment in lung is enhanced in response to bleomycin exposure and ameliorates its fibrotic effects. Proc Natl Acad Sci USA 2003;100:8407-8411.

23. Wagers AJ, Sherwood RI, Christensen JL, et al. Little evidence for developmental plasticity of adult hematopoietic stem cells. Science 2002;297:2256-2259.

24. Ishizawa K, Kubo $\mathrm{H}$, Yamada $\mathrm{M}$, et al. Bone marrow-derived cells contribute to lung regeneration after elastase-induced pulmonary emphysema. FEBS Lett 2004;556:249-252.

25. Zhao YD, Courtman DW, Deng Y, et al. Rescue of monocrotalineinduced pulmonary arterial hypertension using bone marrow-derived endothelial-like progenitor cells: efficacy of combined cell and eNOS gene therapy in established disease. Circ Res 2005;96:442-450.

26. Pittinger MF, Mackay AM, Beck SC, et al. Multilineage potential of adult human mesenchymal stem cells. Science 1999;284:143-147.

27. Jones EA, Kinsey SE, English $A$, et al. Isolation and characterization of bone marrow multipotential mesenchymal progenitor cells. Arthritis Rheum 2002;46:3349-3360.

28. Zvaifler NJ, Marinova-Mutafchieva L, Adams G, et al. Mesenchymal precursor cells in the blood of normal individuals. Arthritis Res 2000;2:477-488.

29. Zuk PA, Zhu M, Ashjian $P$, et al. Human adipose tissue is a source of multipotent stem cells. Mol Biol Cell 2002;13:4279-4295.

30. De Bari D, Dell'Accio F, Tylzanowski P, et al. Multipotent mesenchymal stem cells from adult human synovial membrane. Arthritis Rheum 2001:44:1928-1942.

31. Tuli $R$, Tuli $S$, Nandi $S$, et al. Characterization of multipotential mesenchymal progenitor cells from human trabecular bone. Stem Cells 2003;21:681-693.

32. Barry FP, Murphy JM. Mesenchymal stem cells: clinical applications and bilogical characterization. Int J Biochem Cell Biol 2004;36: 568-584.

33. Simmons PJ, Torok-Storb B. Identification of stromal cell precursors in human bone marrow by a novel monoclonal antibody, STRO1. Blood 1991;78:55-62.

34. Barry FP, Boynton RE, Hanyesworth $\mathrm{S}$, et al. The monoclonal antibody $\mathrm{SH}-2$, raised against human mesenchymal stem cells, recognizes an epitope on endoglin (CD105). Biochem Biophys Res Commun 1999;265:134-139.

35. Barry F, Boynton R, Murphy M, et al. The $\mathrm{SH} 3$ and $\mathrm{SH} 4$ antibodies recognize distinct epitopes on CD73 from human mesenchymal stem cells. Biochem Biophys Res Commun 2001;289:519-524.

36. Krause DS. Engraftment of bone marrow-derived epithelial cells. Ann NY Acad Sci 2005;1044:117-124.

37. Bruscia EM, Ziegler EC, Price JE, et al. Engraftment of donor-derived epithelial cells in multiple organs following bone marrow transplantation into newborn mice. Stem Cells 2006;24:2299-2308.

38. Hennrick KT, Keeton AG, Nanua $S$, et al. Lung cells from neonates show a mesenchymal stem cell phenotype. Am J Respir Crit Care Med 2007;175:1158-1164.

39. Lama VN, Smith L, Badri L, et al. Evidence for tissue-resident mesenchymal stem cells in human adult lung from studies of transplanted allografts. J Clin Invest 2007;117:989-996.
40. Sabatini F, Petecchia L, Tavian M, et al. Human bronchial fibroblasts exhibit a mesenchymal stem cell phenotype and multilineage differentiating potentialities. Lab Invest 2005;85:962-971.

41. Sudo K, Kanno M, Miharada $K$, et al. Mesenchymal progenitors able to differentiate into osteogenic, chondrogenic, and/or adipogenic cells in vitro are present in most primary fibroblast-like cell populations. Stem Cells 2007;25:1610-1617.

42. Summer R, Fitzsimmons $K$, Dwyer $D$, et al. Isolation of an adult mouse lung mesenchymal progenitor cell population. Am J Respir Cell Mol Biol 2007;37:152-159.

43. Ling $\mathrm{T}$, Kuo $\mathrm{M}$, Li C, et al. Identification of pulmonary Oct-4 stem/ progenitor cells and demonstration of their susceptibility to SARS coronavirus (SARS-CoV) infection in vitro. Proc Natl Acad Sci USA 2006;103:9530-9535.

44. Martin J, Helm K, Ruegg P, et al. Adult lung side population cells have mesenchymal stem cell potential. Cytotherapy 2008;10:140-115.

45. Goodell MA, Brose K, Paradis $G$, et al. Isolation and functional properties of murine hematopoietic stem cells that are replicating in vivo. J Exp Med 1996;183:1797-1806.

46. Kim YJ, Yu JM, Joo HJ, et al. Role of CD9 in proliferation and proangiogenic action of human adipose-derived mesenchymal stem cells. Eur J Physiol 2007;455:283-296.

47. Levy $S$, Shoham T. Protein-protein interactions in the tetraspanin web. Physiology 2005;20:218-224.

48. Hemler ME. Tetraspanin functions and associated microdomains. Nat Rev Mol Cell Biol 2005;6:801-811.

49. Maecker HT, Todd SC, Levy S. The tetraspanin superfamily: molecular facilitators. FASEB J 1997;11:428-442.

50. Boucheix C, Rubinstein E. Tetraspanins. Cell Mol Life Sci 2001;58:1189-1205.

51. Aoyama K, Oritani K, Yokota T, et al. Stromal cell CD9 regulates differentiation of hematopoietic stem/progenitor cells. Blood 1999;93:2586-2594.

52. Seehafer JG, Shaw AR. Evidence that the signal-initiating membrane protein CD9 is associated with small GTP-binding proteins. Biochem Biophys Res Commun 1991;179:401-406.

53. Nakamura $\mathrm{Y}$, Handa $\mathrm{K}$, Iwamoto $\mathrm{R}$, et al. Immunohistochemical distribution of $C D 9$, heparin binding epidermal growth factor-like growth factor, and integrin alpha3beta1 in normal human tissues. J Histochem Cytochem 2001;49:439-444.

54. Funakoshi T, Tachibana I, Hoshida Y, et al. Expression of tetraspanins in human lung cancer cells: frequent downregulation of CD9 and its contribution to cell motility in small cell lung cancer. Oncogene 2003;22:674-687.

55. Higashiyama $\mathrm{M}$, Doi $\mathrm{O}$, Kodama $\mathrm{K}$, et al. Immunohistochemically detected expression of motility-related protein-1 (MRP-1/CD9) in lung adenocarcinoma and its relation to prognosis. Int J Cancer 1997;74:205-211.

56. Samadikuchaksaraei A, Bishop AE. Derivation and characterization of alveolar epithelial cells from murine embryonic stem cells in vitro. Methods Mol Biol 2006;330:233-248.

57. Samadikuchaksaraei A, Bishop AE. Effects of growth factors on the differentiation of murine ESC into type II pneumocytes. Cloning Stem Cells 2007;9:407-416.

58. Sueblinvong $\mathrm{V}$, Loi $\mathrm{R}$, Eisenhauer $\mathrm{PL}$, et al. Derivation of lung epithelium from human cord blood-derived mesenchymal stem cells. Am J Respir Crit Care Med 2008;177:701-711.

59. Fehrenbach $\mathrm{H}$. Alveolar epithelial type II cell: defender of the alveolus revisited. Respir Res 2001;2:33-46.

60. Danto SI, Shannon JM, Borok Z, et al. Reversible transdifferentiation of alveolar epithelial cells. Am J Respir Cell Mol Biol 1995;12:497-502.

61. Bui KC, Buckley S, Wu F, et al. Induction of A- and D-type cyclins and cdc kinase activity during recovery from short term hypertoxic lung injury. Am J Physiol 1995;268:L625-L635.

62. Wang J, Edeen K, Manzer R, et al. Differentiated human alveolar epithelial cells and reversibility of their phenotype in vitro. Am J Respir Cell Mol Biol 2007;36:661-668.

63. Wuenschell CW, Sunday ME, Singh G, et al. Embryonic mouse lung epithelial progenitor cells co-express immunohistochemical markers of diverse mature cell lineages. J Histochem Cytochem 1996;44:113-123.

64. Meneghetti A, Cardoso WV, Brody JS, et al. Epithelial marker genes are expressed in cultured embryonic rat lung and in vivo with similar spatial and temporal patterns. J Histochem Cytochem 1996;44: 1173-1182. 
65. Liebler JM, Lutzko C, Banfalvi A, et al. Retention of human bone marrow-derived cells in murine lungs following bleomycin-induced lung injury. Am J Physiol Lung Cell Mol Physiol 2008;285: L285-L292.

66. Wong AP, Keating A, Lu WY, et al. Identification of a bone marrowderived epithelial-like population capable of repopulating injured mouse airway epithelium. J Clin Invest 2009;119:336-348.

67. Mattsson J, Jansson M, Wernerson A, et al. Lung epithelial cells and type II pneumocytes of donor origin after allogeneic hematopoietic stem cell transplantation. Transplantation 2004;78:154-157.

68. Suratt BT, Cool CD, Serls AE, et al. Human pulmonary chimerism after hematopoietic stem cells transplantation. Am J Resp Crit Care Med 2003;168:318-322.
69. Goodell MA. Multipotential stem cells and 'side population' cells. Cytotherapy 2002;4:507-508.

70. Challen GA, Little MH. A side order of stem cells: the SP phenotype. Stem Cells 2006;24:3-12.

71. Majka SM, Beutz MA, Hagen $M$, et al. Identification of novel resident pulmonary stem cells: form and function of the lung side population. Stem Cells 2005;23:1073-1081.

72. Staud F, Pavek P. Breast cancer resistance protein (BCRP/ABCG2). Int J Biochem Cell Biol 2005;37:720-725.

73. Doyle LA, Ross DD. Multidrug resistance mediated by the breast cancer resistance protein BCRP (ABCG2). Oncogene 2003;22:7340-7358.

74. Polgar O, Robey RW, Bates SE. ABCG2: structure, function and role in drug response. Expert Opin Drug Metab Toxicol 2008;4:1-15. 